related injury and disability are well-known. However, the characteristics of workers who have multiple workers' compensation claims in a defined period of time is less clearly understood. This study estimates the extent to which industries, occupations or injury characteristics are associated with a higher risk for the registration of repeat claims. We present a parallel analysis of Ontario, Canada, and Victoria, Australia to determine if there aredifferences in repeat claim patterns between these two jurisdictions.

Methods First lost-time claims of greater than ten days of wagereplacement benefits were identified in the period January 2000 to December 2004 using administrative records of workers' compensation claims. Claimants were followed prospectively for subsequent claims for 5 years from the date of the first claim to estimate the risk for a repeat claim by industry, occupation and injury characteristics.

Results The five year cumulative incidence of an initial claim in Victoria (14.5/1000 person years) was twice the rate observed in Ontario (7.6/1000 person years) The probability of a second lost-time claim of ten days or longer duration within 5 years of the date of the first injury was 0.25 in Victoria and 0.12 in Ontario. The probability of a second claim was related to age, sex, nature of injury, occupation and industry. The distribution of characteristics of second injuries was similar between Ontario and Victoria.

Conclusion This study has used population-based work disability insurance records to compare the incidence of repeat compensation claims in two jurisdictions. Despite differences in scheme benefit policies, this study has found broadly similar patterns in the two settings. The potential for causal inference regarding risk factors for repeat claims is discussed.

\section{RESULTS OF A MYOTONOMETRICAL MUSCLE STUDY IN PATIENTS WITH WORK-RELATED HAND OVERLOAD DISORDER}

${ }^{1} V$ P Pille, ${ }^{2}$ Oha, ${ }^{1}$ Tint. ' Tallinn University of Technology, Tallinn, Estonia; ${ }^{2}$ Tartu University, Tartu, Estonia

\subsection{6/oemed-2013-101717.133}

Introduction Implementation of the new myotonometrical diagnostic method in occupational medicine enables non-invasive, easy and quick evaluation of the functional state of muscles.

Aims Use of the myotonometrical method to study hand muscles in patients with work-related hand overload disorders, and analysing the correlations of the results of myotonometrical testing broken down by various diagnosed disease groups. To analyse whether myotonometry could be used in occupational medicine as a method for evaluating the functional state of muscles.

Method 25 patients with diagnosed work related carpal tunnel syndrome or/and tenosynovitis in the right wrist were studied. Diseases were diagnosed using ENMG and ultrasound examinations.

The mechanical characteristics of frequency, stiffness and elasticity of right hand muscle $m$. abductor pollicis brevis were recorded by means of the Myoton-3 hand-held myotonometer.

For data processing, the Stata program and the Student T-test were used.

Results An average age of patients 54, standard deviation 6.6, $\min 42$ and max 67 , the length of service 24.8 years, the average workday length 6.8 hours. Significant changes regarding muscle decrement and stiffness were apparent in affected workers as compared to the norm.

In subjects with the diagnosis of abd poll br decrement vs norm, the average values were 2.0 and $1.6, \mathrm{p}<0.001$; i.e. there was a significant difference. Stiffness differed significantly from the norm: 311.4 vs $348.8, \mathrm{p}=0.004$.

Conclusion In case of carpal tunnel syndrome and tenosynovitis due to overload of the right wrist area, also signs of an overload of the abductor pollcis muscle can be detected by myotonometric testing. Consequently, myotonometrical muscle study could be used as an auxiliary diagnostic method as well as an initial study in the course of physical examination in order to evaluate possible hand muscle overload.

\section{VARIABILITY OF WORK-RELATED MUSCULOSKELETAL SYMPTOMS, WORK EXPOSURES, AND CONDITIONS BY EMPLOYMENT SETTING AMONG UNITED STATES CERTIFIED ATHLETIC TRAINERS}

${ }^{1}$ L Kucera, 'Lipscomb, 'Dement, ${ }^{2}$ Hootman, ${ }^{3}$ Roos. ${ }^{1}$ Duke University Medical Center, Durham, United States of America; ${ }^{2}$ Centers for Disease Control and Prevention, Atlanta, GA, United States of America; ${ }^{3}$ University of North Carolina, Chapel Hill, NC, United States of America

\subsection{6/oemed-2013-101717.134}

Objectives In addition to colleges and schools, certified athletic trainers (ATs) are now found in hospitals, clinics and other settings including industry. We describe ATs musculoskeletal symptoms, and work exposures and conditions in different settings.

Methods We administered online surveys to a random sample of 10,000 US ATs. Participants $(n=1826)$ estimated the frequency they performed selected work tasks and rated task-related stress [scale 0 (no problem) to 10 (major problem)]. Musculoskeletal symptoms (MS) that interfered or prevented work in the last year was reported by body region as well as frequency with which ten protective strategies were used to reduce work-related physical strain [scale 1 (never) to 5 (always)].

Results ATs commonly worked in schools (67\%) and clinics or hospitals (20\%). Prevalence of MS that interfered/prevented work was $27.2 \%$ (95\% CI: 25.1\%, 29.3\%); prevalence was highest in the low back (13.7\%) and wrist/hand (7.0\%). Back pain was higher in schools $(14.7 \%)$ and other (12.9\%) settings compared to clinics/hospitals (9.7\%). ATs in schools more frequently performed taping/wrapping, used treatment modalities, handled/moved equipment, cared for multiple patients at once, worked under time constraints, awkward positions or sustained positions, and inclement weather conditions. School ATs rated physical stress of handling/moving equipment (mean $=4.9$ ), working in same position (4.8), inclement weather conditions (4.0), being short staffed (5.0) and work scheduling issues (5.4) higher than clinic/hospital ATs. Mental stress due to understaffing (mean $=4.9$ ), increased workload (5.0), irregular schedules (5.1), and long work weeks (5.8) were also rated higher by school ATs. School ATs reported always or almost always getting help with patient transfers, asking others to perform physically stressful tasks, and using electrotherapy instead of manual techniques to avoid injury aggravation.

Conclusions Work tasks, physical and mental stressors, and use of protective strategies varied by work setting. Preventive measures need to consider variability across work settings. 